\title{
Adjustment disorders: prevalence in a representative nationwide survey in Germany
}

\author{
Maercker, Andreas ; Forstmeier, Simon ; Pielmaier, Laura ; Spangenberg, Lena ; Brähler, Elmar ;
} Glaesmer, Heide

\begin{abstract}
OBJECTIVES: This is the first study to estimate the prevalence of adjustment disorder (AjD) in the general population. A new conceptualisation of $\mathrm{AjD}$ as a stress response syndrome was applied, which allowed $\mathrm{AjD}$ to be assessed directly from its symptom profile, including intrusive, avoidance and failure-to-adapt symptoms (Maercker et al., Psychopathology 40:135-146, 2007). METHODS: Prevalence rates of distressing life events and $\mathrm{AjD}$ were estimated from a representative sample of the German general population $(\mathrm{n}=2,512)$ with a broad age range (14-93 years). A questionnaire including a life events checklist and self-rating questions that assessed AjD symptoms and symptom duration were personally handed out by an interviewer. RESULTS: The prevalence of AjD fulfilling the criterion of clinically significant impairment was $0.9 \%$; a further $1.4 \%$ of the sample was diagnosed with AjD without fulfilling the impairment criterion. In $72.5 \%$ of $\mathrm{AjD}$ cases, symptoms had developed 6-24 months prior to assessment. AjD was most often associated with acute events such as moving or chronic stressors such as serious illness, conflicts at the respondent's job or with friends or neighbours (with $5 \%$ conditional probability each). CONCLUSIONS: The results correspond with the few other studies that have examined the prevalence of $\mathrm{AjD}$, even though a new conceptualisation of the disorder was used. Explorative results regarding the duration of $\mathrm{AjD}$ syndromes and symptoms call for further redefinition and empirical investigation of this under-researched mental condition.
\end{abstract}

DOI: https://doi.org/10.1007/s00127-012-0493-x

Posted at the Zurich Open Repository and Archive, University of Zurich

ZORA URL: https://doi.org/10.5167/uzh-61447

Journal Article

Published Version

Originally published at:

Maercker, Andreas; Forstmeier, Simon; Pielmaier, Laura; Spangenberg, Lena; Brähler, Elmar; Glaesmer, Heide (2012). Adjustment disorders: prevalence in a representative nationwide survey in Germany. Social Psychiatry and Psychiatric Epidemiology, 47(11):1745-1752.

DOI: https://doi.org/10.1007/s00127-012-0493-x 


\title{
Adjustment disorders: prevalence in a representative nationwide survey in Germany
}

\author{
Andreas Maercker - Simon Forstmeier - Laura Pielmaier • \\ Lena Spangenberg • Elmar Brähler · Heide Glaesmer
}

Received: 12 September 2011/ Accepted: 25 February 2012

(C) Springer-Verlag 2012

\begin{abstract}
Objectives This is the first study to estimate the prevalence of adjustment disorder (AjD) in the general population. A new conceptualisation of AjD as a stress response syndrome was applied, which allowed AjD to be assessed directly from its symptom profile, including intrusive, avoidance and failure-to-adapt symptoms (Maercker et al., Psychopathology 40:135-146, 2007).

Methods Prevalence rates of distressing life events and $\mathrm{AjD}$ were estimated from a representative sample of the German general population $(n=2,512)$ with a broad age range (14-93 years). A questionnaire including a life events checklist and self-rating questions that assessed AjD symptoms and symptom duration were personally handed out by an interviewer.

Results The prevalence of AjD fulfilling the criterion of clinically significant impairment was $0.9 \%$; a further $1.4 \%$ of the sample was diagnosed with AjD without fulfilling the impairment criterion. In $\sim 72.5 \%$ of $\mathrm{AjD}$ cases, symptoms had developed 6-24 months prior to assessment. $\mathrm{AjD}$ was most often associated with acute events such as moving or chronic stressors such as serious illness, conflicts at the respondent's job or with friends or neighbours (with $\sim 5 \%$ conditional probability each).

Conclusions The results correspond with the few other studies that have examined the prevalence of $\mathrm{AjD}$, even though a new conceptualisation of the disorder was used.
\end{abstract}

\footnotetext{
A. Maercker $(\bowtie) \cdot$ S. Forstmeier $\cdot$ L. Pielmaier Department of Psychology, University of Zurich, Binzmuhlestr. 14, 8050 Zurich, Switzerland e-mail: maercker@psychologie.uzh.ch

L. Spangenberg · E. Brähler $\cdot$ H. Glaesmer Department of Medical Psychology and Medical Sociology, University of Leipzig, Leipzig, Germany
}

Explorative results regarding the duration of AjD syndromes and symptoms call for further redefinition and empirical investigation of this under-researched mental condition.

Keywords Adjustment disorders - Stress-response syndromes $\cdot$ Survey $\cdot$ Nationwide study

\section{Introduction}

In preparing the DSM-5 and ICD-11, new epidemiological data on adjustment disorder (AjD) are required. Because of yet unresolved validity problems, there is an increased research interest in AjD. These problems concern the conceptualisation of the disorder itself, the validity of the exclusion criterion ("does not meet the criteria for another specific disorder"), the time criterion ("the symptoms do not persist for more than an additional 6 months"), and the definition of subgroups [1-3]. At the same time, many good reasons justify the use of the diagnosis in clinical practice [4]. For example, therapeutic strategies used to treat $\mathrm{AjD}$, like problem solving, differ from treatments for depression or anxiety disorders $[5,6]$.

To overcome problems in defining AjD, Maercker et al. [7] proposed a new diagnostic model that conceptualises $\mathrm{AjD}$ as a stress response syndrome, similar to acute or posttraumatic stress disorder (PTSD) but without the necessity of a previously experienced traumatic or lifethreatening event (see "Appendix"). In contrast to PTSD, index events of AjD are single or ongoing severe life events (e.g. divorce, illness, job loss, conflicts at work) or other stressors (ongoing psychosocial difficulty or a combination of stressful life conditions such as family conflicts, moving from one place to another or particular refugee conditions). 
A series of studies has demonstrated the internal, convergent and discriminant validity of the new model. A populationbased study with older individuals revealed a point prevalence of $2.3 \%$ [8]. In refugee camps on different continents, prevalence rates of $\mathrm{AjD}$ ranged from 6 (Ethiopia) to $40 \%$ (Algeria) because of a loss of property, lack of shelter or forced separation from family [9]. The stress response concept has been recognised by the DSM-5 committee, and they plan to adopt it in the DSM-5 [10]. A further recommendation to delete the previous exclusion criterion to allow for the simultaneous diagnosis of other Axis I disorders [2] is also gaining increasing acceptance with the DSM-5 committee. Also, the corresponding ICD-11 working group considers the new diagnostic model for reformulating AjD [11]. Both classification systems have not decided how to deal with the impairment criterion for $\mathrm{AjD}$ in the future.

Thus far, studies on $\mathrm{AjD}$ prevalence in the general population are rare. The only large-scale multisite investigation that exists reported a point prevalence of $1 \%$ [12]. Diagnoses were established applying the exclusion criterion ("does not meet the criteria for another specific disorder"). Most other epidemiological data on AjD are from primary care and consultation-liaison psychiatry where prevalence rates varied between 7 and 34\% [4, 13, 14].

This paper concerns the prevalence of $\mathrm{AjD}$ according to the new stress response syndrome model [7], which requires (1) the presence of one or more identifiable stressors, (2) the three constitutive symptom clusters, namely, intrusive symptoms (e.g. involuntary stressful reminders), avoidance behaviour (e.g. attempts not to talk about the event) and symptoms of failure to adapt (e.g. avoidance of feelings about the event), and (3) additional characteristics determining the subtype (e.g. depressed, anxious, impulsive). Additionally, we asked for the duration of symptom persistence. Only stressful events and symptoms that occurred during the past 2 years were considered.

The main aim of this study is to estimate the prevalence of AjD within a population-based sample. Additionally, the frequency of index stressors will be assessed, which allows for calculating conditional probabilities of each stressor category. Since few available data exist on gender and age group distribution [12], basic analyses will include these aspects. Finally, we present quantitative and qualitative data on the duration of $\mathrm{AjD}$.

\section{Methods}

\section{Participants}

A representative sample of the German general population was selected with the assistance of a demographic consulting company (USUMA, Berlin, Germany). Germany was divided into 258 sample areas corresponding to the different regions of the country. After a sample area was selected, households in that area were selected by the random route procedure. One member of each household fulfilling the study's inclusion criteria (i.e. at least 14 years old and able to read and understand the German language) was selected randomly by the Kish selection grid technique. This technique is used for sampling individuals on the doorstep from among household residents. The system is devised so that all individuals in a household have an equal chance of selection. A first interview attempt was made for 4,630 addresses, of which 4,572 were valid. If the resident was not at home, a maximum of three attempts was made to contact the selected person. Overall, 1,546 persons $(33.8 \%)$ declined to participate, 497 subjects $(10.9 \%)$ were not reached after three attempts, and 5 persons $(0.1 \%)$ refused participation because of severe health problems. All participants were visited by a study assistant, informed about the investigation and presented with selfrating questionnaires. Participants gave their informed consent prior to the assessment. The assistant waited until the participant answered all questionnaires and offered help if the participant did not understand the items.

The data collection took place in May and June 2009. A total of 2,524 people agreed to participate and completed the self-rating questionnaires (participation rate: $55.2 \%$ of valid addresses). Despite the moderate size of the response rate, the sample was representative of the German general population in its distribution of demographic characteristics (Statistisches Bundesamt, 2009). Therefore, all analyses were conducted using unweighted data.

The study was conducted following the ethical standards of the German medical and psychological associations. Formal approval of the study was not necessary, as strict standards of confidentiality, voluntariness and respondent protection were observed.

Participants were between 14 and 93 years old with an average age of 49.6 years (SD 17.9) (see Table 1); $55.8 \%$ were female and $44.2 \%$ were male. For further specific analyses, respondents were divided into three age groups: 14-29 years ("young adults"), 30-59 years ("middle-aged adults") and 60-95 years ("older adults").

\section{Assessments}

\section{AjD stressor list}

Seven types of acute events (e.g. divorce, moving; see Table 2) and ten types of chronic stressors (e.g. conflict with neighbours, serious illness) were assessed if they occurred during the last 2 years by endorsing 'yes' or 'no' [8]. Additionally, three open-ended questions asked for other events ("Other stressors? Please specify."). Answers 
Table 1 Sample characteristics $(N=2,512)$

\begin{tabular}{|c|c|c|}
\hline & $n$ & $\%$ \\
\hline \multicolumn{3}{|l|}{ Gender } \\
\hline Female & 1,401 & 55.77 \\
\hline Male & 1,111 & 44.23 \\
\hline \multicolumn{3}{|l|}{ Age (years) } \\
\hline $14-29$ & 432 & 17.20 \\
\hline $30-59$ & 1,258 & 50.08 \\
\hline $60-93$ & 822 & 32.72 \\
\hline \multicolumn{3}{|l|}{ Marital status/partnership } \\
\hline Married & 1,264 & 50.32 \\
\hline Single & 652 & 25.96 \\
\hline Divorced & 290 & 11.54 \\
\hline Widowed & 306 & 12.18 \\
\hline Living with partner & 1,403 & 55.85 \\
\hline Not living with partner & 1,109 & 44.15 \\
\hline \multicolumn{3}{|l|}{ Education } \\
\hline Elementary school & 1,189 & 47.33 \\
\hline Secondary school & 971 & 38.65 \\
\hline Higher education & 352 & 14.01 \\
\hline \multicolumn{3}{|l|}{ Employment } \\
\hline In training & 171 & 6.81 \\
\hline Full- or part-time job & 1,230 & 48.96 \\
\hline Homemaker or similar & 152 & 6.05 \\
\hline Unemployed & 195 & 7.76 \\
\hline Retired & 764 & 30.41 \\
\hline
\end{tabular}

to the open-ended questions that did not fit into the specified categories were combined into a residual category.

In this section, respondents were instructed to indicate all severe events that they had experienced (the seven acute events and ten chronic stressors they had experienced in the last 2 years: 'yes' or 'no'), disregarding the amount of subjective distress each event caused. Subsequently, they were asked to specify which three events caused them the most subjective distress. For the following calculations, the stressor mentioned first was considered to be the most distressing event. Additionally, participants indicated the time that had passed since the event, or if the event was still ongoing, and the time period for which the event had been occurring $(<1$ month, 1-6 months, 6-24). The 2-year limit was employed according to the ICD-10 limit for adjustment disorders to 24 months (whereas DSM-IV lacks such time limitation).

\section{AjD criteria}

The AjD questionnaire [15] was shortened from 29 to 19 items. Item selection for the abridged version of the questionnaire was based on previous factor analytic studies that examined the structure of the questionnaire [7, 15]. Additionally, a criterion of impairment was assessed, according to the DSM-IV definition of mental disorders: "The symptoms cause clinically significant impairment in social, occupational, or other important areas of functioning" (possible responses were 1 [none]-4 [most]).

Symptom criteria endorsement and onset of symptoms were assessed with regard to the most distressing AjD life event specified before. Core symptom clusters (intrusions, four items; avoidance, four items; and failure to adapt, three items) and subtypes (depressed, three items; anxiety, two items; and impulse disturbance, three items) were assessed. Participants indicated the frequency of these items on a 4-point Likert scale (1, never; 2, rarely; 3, sometimes; 4 , often). In addition, they were asked to indicate the duration of symptom occurrence: $<1$ month, 1-6 months or 6-24 months.

\section{AjD diagnoses and subtypes}

AjD was diagnosed if all three core symptom groups (intrusions, avoidance and failure to adapt) were present with the numerical majority of symptom frequency ratings $\geq 3$ or a symptom cluster-specific comprehensive value score $\geq 9$. Accordingly, AjD subtypes were defined: depressed, disturbance of conduct (or: impulsive disturbance) and mixed subtypes were diagnosed if the numerical majority of symptom values was $\geq 3$; anxiety subtype (two items) was diagnosed if at least one symptom was $\geq 3$.

$\mathrm{AjD}$ was diagnosed as either fulfilling or not fulfilling the impairment criterion (for the former, the impairment criterion value was required to be $\geq 3$ ). No other clinical diagnoses were assessed.

\section{Results}

The prevalence of AjD life events is displayed in Table 2 categorised by acute events versus chronic stressors. Note that multiple answers were allowed. In total, $53.9 \%$ of the respondents reported at least one $\mathrm{AjD}$ distressing life event. The subgroup of participants that experienced at least one stressor indicated on average 1.7 (SD 1.1) life events. The rightmost column in Table 2 shows the life events that participants reported as being the most severe, which were considered to be index stressors for the purposes of determining associated distress in subsequent analyses.

Independent of how many life events respondents mentioned and their rated severity, the most frequently mentioned AjD life events were death of a loved one and financial difficulties. These were followed in frequency by job loss, job-related conflict and family conflict. Significant gender differences were found for the life events of 
Table 2 Lifetime prevalence rates of severe life events (LE) by gender $(N=2,512)$

\begin{tabular}{|c|c|c|c|c|c|c|c|c|c|}
\hline & \multicolumn{2}{|c|}{ Total } & \multicolumn{2}{|c|}{ Female } & \multicolumn{2}{|c|}{ Male } & \multirow{2}{*}{$\begin{array}{l}\text { Gender differences } \\
\chi^{2}\end{array}$} & \multicolumn{2}{|c|}{ Most severe LE } \\
\hline & $n$ & $\%$ & $n$ & $\%$ & $n$ & $\%$ & & $n$ & $\%$ \\
\hline \multicolumn{10}{|l|}{ Acute events } \\
\hline Death of a loved one & 371 & 14.77 & 211 & 15.06 & 160 & 14.40 & 0.21 & 243 & 9.67 \\
\hline Moving & 169 & 6.73 & 105 & 7.49 & 64 & 5.76 & 2.97 & 22 & 0.88 \\
\hline Divorce & 102 & 4.06 & 70 & 5.00 & 32 & 2.88 & $7.12 * *$ & 66 & 2.63 \\
\hline Termination of leisure activity & 41 & 1.63 & 21 & 1.50 & 20 & 1.80 & 0.35 & 9 & 0.36 \\
\hline Retirement & 41 & 1.63 & 18 & 1.28 & 23 & 2.07 & 2.38 & 13 & 0.52 \\
\hline Accident & 38 & 1.51 & 24 & 1.71 & 14 & 1.26 & 0.85 & 14 & 0.56 \\
\hline Criminal act (e.g. burglary) & 10 & 0.40 & 4 & 0.29 & 6 & 0.54 & 1.01 & 5 & 0.20 \\
\hline \multicolumn{10}{|l|}{ Chronic stressors } \\
\hline Financial difficulties & 258 & 10.27 & 139 & 9.92 & 119 & 10.71 & 0.42 & 125 & 4.98 \\
\hline Too much or too little work & 249 & 9.91 & 130 & 9.28 & 119 & 10.71 & 1.42 & 63 & 2.51 \\
\hline Family conflicts & 181 & 7.21 & 118 & 8.42 & 63 & 5.67 & $7.02 * *$ & 98 & 3.90 \\
\hline Illness/care for a loved one & 174 & 6.93 & 114 & 8.14 & 60 & 5.40 & $7.20 * *$ & 95 & 3.78 \\
\hline Unemployment & 162 & 6.45 & 82 & 5.85 & 80 & 7.20 & 1.87 & 92 & 3.66 \\
\hline Pressure to meet deadlines & 146 & 5.81 & 73 & 5.21 & 73 & 6.57 & 2.09 & 63 & 2.51 \\
\hline Serious illness & 132 & 5.25 & 84 & 6.00 & 48 & 4.32 & 3.49 & 92 & 3.66 \\
\hline Conflicts at job & 98 & 3.90 & 43 & 3.07 & 55 & 4.95 & 5.85 & 39 & 1.55 \\
\hline Conflicts with neighbours & 89 & 3.54 & 54 & 3.85 & 35 & 3.15 & 0.90 & 36 & 1.43 \\
\hline Other & 43 & 1.71 & 32 & 2.28 & 11 & 0.99 & $6.17 *$ & 29 & 1.15 \\
\hline
\end{tabular}

For gender differences, $* p<0.05, * * p<0.01$

divorce, family conflict and illness/care of a loved one, as well as for the residual category. In all cases, women indicated these categories more often than men, as indicated by $\chi^{2}$ testing (Table 2).

Table 3 presents the prevalence rates of AjD symptom clusters and AjD diagnoses. Clinically relevant symptoms (i.e. those that exceeded the cutoff score) were predominantly intrusion symptoms; $9.1 \%$ of the total sample experienced these, which corresponds to $16.9 \%$ of the participants reporting at least one AjD stressor event. A total of $1.4 \%$ of the sample was diagnosed with $\mathrm{AjD}$ without fulfilling the impairment criterion, and $0.9 \%$ was diagnosed with AjD fulfilling the impairment criterion. Even though all core symptom groups showed substantial gender differences (women $>$ men), no significant gender differences were found within the two AjD diagnosis groups. Prevalence rates of AjD without clinical impairment by age group were $0.2 \%(n=1)$ for young adults, $1.7 \%(n=22)$ for middle-aged adults and $1.3 \%(n=11)$ for older adults $\left(\chi^{2}=5.55 ; p=0.06\right)$.

The prevalence of all subtype characteristic symptoms (depressive, anxious and conduct disturbance) ranged from 3.7 to $4.4 \%$ and was fairly comparable across the total sample. Consequently, mixed subtypes were overrepresented; the subtype most frequently associated with a diagnosis of $\mathrm{AjD}$ without impairment was mixed disturbance of emotions and conduct $(n=11 ; 0.04 \%)$ followed by mixed anxiety and depressed $\operatorname{mood}(n=4 ; 0.02 \%)$ and other mixed types $(n=8 ; 0.03 \%)$. By contrast, singlefeature subtypes were less common: $n=4(0.02 \%)$ for AjD with depressed mood; $n=3(0.01 \%)$ for AjD with impulse disturbance and $n=2(0.01 \%)$ for $\mathrm{AjD}$ with anxiety.

For most $\mathrm{AjD}$ cases $(72.5 \%)$, the duration of core and subtype characteristic symptom groups was 6-24 months. Another $22 \%$ indicated symptom periods of $1-6$ months, and $5.5 \%$ reported symptom persistence for $<1$ month ( $\chi^{2}$ tests showed significant effects for 5 of the 6 symptom groups; see Table 3).

Furthermore, we calculated conditional probabilities (CP) for each $\mathrm{AjD}$ life event category to estimate the impact of each event. The CP for each type of acute and chronic stressor was calculated (Table 4). The average CP for AjD with any of the listed events was $4.02 \%$. With respect to CPs for each particular life event category, no event type exceeded a $\mathrm{CP}$ of $6 \%$; this result indicates that $<1$ person out of 15 developed AjD after exposure to any specific type of life event. The event types most frequently associated with $\mathrm{AjD}$ were serious illness, conflict with neighbours and job-related conflicts.

Because only a small number of participants were diagnosed with $\mathrm{AjD}$, and because participants reported the duration of each symptom separately, the duration of symptoms for each event category can only be analysed at 
Table 3 Prevalence rates of adjustment disorder symptoms and subtypes, and overall by gender and duration of symptoms

\begin{tabular}{|c|c|c|c|c|c|c|c|c|c|c|c|c|c|c|}
\hline & \multirow{2}{*}{\multicolumn{2}{|c|}{$\begin{array}{l}\text { Total } \\
(N=2,512)\end{array}$}} & \multicolumn{5}{|c|}{ Gender differences } & \multicolumn{7}{|c|}{ Differences in duration $^{\mathrm{a}}$} \\
\hline & & & \multicolumn{2}{|c|}{$\begin{array}{l}\text { Female } \\
(n=1,401)\end{array}$} & \multicolumn{2}{|c|}{$\begin{array}{l}\text { Male } \\
(n=1,111)\end{array}$} & \multirow[t]{2}{*}{$\chi^{2}$} & \multicolumn{2}{|c|}{$<1$ month } & \multicolumn{2}{|c|}{$1-6$ months } & \multicolumn{2}{|c|}{$6-24$ months } & \multirow[t]{2}{*}{$\chi^{2}$} \\
\hline & $n$ & $\%$ & $n$ & $\%$ & $n$ & $\%$ & & $n$ & $\begin{array}{l}\text { cond. } \\
(\%)^{\mathrm{b}}\end{array}$ & $n$ & $\begin{array}{l}\text { cond. } \\
(\%)^{\mathrm{b}}\end{array}$ & $n$ & $\begin{array}{l}\text { cond. } \\
(\%)^{\mathrm{b}}\end{array}$ & \\
\hline Intrusions & 229 & 9.1 & 155 & 11.1 & 74 & 6.7 & $14.50 * * *$ & 11 & 5.1 & 50 & 23.3 & 154 & 71.6 & $12.07 * *$ \\
\hline Avoidance & 137 & 5.5 & 90 & 6.4 & 47 & 4.2 & $5.78 *$ & 6 & 4.8 & 33 & 26.4 & 86 & 68.8 & $9.10^{* *}$ \\
\hline Failure to adapt & 97 & 3.9 & 71 & 5.1 & 26 & 2.3 & $12.42 * * *$ & 3 & 3.3 & 21 & 22.8 & 68 & 73.9 & $15.24 * * *$ \\
\hline Depressed & 111 & 4.4 & 79 & 5.6 & 32 & 2.9 & $11.16 * * *$ & 2 & 1.9 & 21 & 19.8 & 83 & 78.3 & $11.57 * *$ \\
\hline Anxious & 93 & 3.7 & 63 & 4.5 & 30 & 2.7 & $5.61 *$ & 4 & 4.4 & 21 & 23.1 & 66 & 72.5 & $14.41 * * *$ \\
\hline Impulsive & 94 & 3.7 & 56 & 4.0 & 38 & 3.4 & 0.57 & 10 & 10.9 & 18 & 19.6 & 64 & 69.6 & 4.32 \\
\hline AjD with impairment & 23 & 0.9 & 16 & 1.1 & 7 & 0.6 & 1.79 & 1 & 4.8 & 3 & 14.3 & 17 & 81.0 & 3.61 \\
\hline $\begin{array}{l}\mathrm{AjD} \text { without } \\
\text { impairment }\end{array}$ & 34 & 1.4 & 22 & 1.6 & 12 & 1.1 & 1.12 & 1 & 3.3 & 5 & 16.7 & 24 & 80.0 & 4.67 \\
\hline
\end{tabular}

Table 4 Conditional probabilities (CP) of adjustment disorder by type of event

\begin{tabular}{|c|c|c|c|}
\hline & \multicolumn{2}{|c|}{$\mathrm{CP}$ for $\mathrm{AjD}$} & \multirow[t]{2}{*}{ Qualitative/single-case analyses of symptom duration } \\
\hline & $n$ & $\%^{\mathrm{a}}$ & \\
\hline \multicolumn{4}{|l|}{ Acute events } \\
\hline Death of a loved one & 9 & 3.7 & $6-24$ months in most cases and for most of the symptoms \\
\hline Moving & 1 & 4.5 & 6-24 months for most of the symptoms; linked with family conflict and serious illness \\
\hline Divorce & 0 & 0 & \\
\hline Termination of leisure activity & 0 & 0 & \\
\hline Retirement & 0 & 0 & \\
\hline Accident & 0 & 0 & \\
\hline Criminal act (e.g. burglary) & 1 & 2.9 & 6-24 months for most of the symptoms; linked with illness of a loved one \\
\hline \multicolumn{4}{|l|}{ Chronic stressors } \\
\hline Financial difficulties & 5 & 4.0 & $6-24$ months in most cases and for most of the symptoms \\
\hline Too much or too little work & 0 & 0 & \\
\hline Family conflicts & 3 & 3.1 & 6-24 months for most of the symptoms; linked with serious illness for one individual \\
\hline Illness/care for a loved one & 1 & 1.1 & $6-24$ months for most of the symptoms \\
\hline Unemployment & 2 & 2.2 & $6-24$ months for most of the symptoms \\
\hline Pressure to meet deadlines & 2 & 3.2 & 6-24 months for most of the symptoms; linked with financial difficulties \\
\hline Serious illness & 5 & 5.4 & $\begin{array}{l}\text { One individual indicated } 1-6 \text { months for most of the symptoms; in all other cases, } \\
6-24 \text { months for most of the symptoms }\end{array}$ \\
\hline Conflicts at job & 2 & 5.1 & $\begin{array}{l}\text { One individual indicated } 1-6 \text { months for most of the symptoms, and the other } \\
\text { individual indicated 6-24 months for most of the symptoms }\end{array}$ \\
\hline Conflicts with neighbours & 2 & 5.6 & $\begin{array}{l}\text { One individual indicated } 1-6 \text { months for most of the symptoms, and the other } \\
\text { individual indicated 6-24 months for most of the symptoms }\end{array}$ \\
\hline Other & 2 & 3.4 & \\
\hline
\end{tabular}

${ }^{a}$ In relation to the number of participants of the total sample indicating the respective event as their most severe life event (see Table 2)

the level of individual cases (Table 4, right column). To account for this limitation, the second and third life events indicated by respondents were also included. Accordingly, 29 out of 34 participants with AjD diagnoses reported that most symptoms persisted for 6-24 months, whereas only 6 respondents indicated durations of $\mathrm{AjD}$ symptoms for less than 6 months. Five individuals who indicated that their AjD symptoms lasted for 6-24 months noted multiple life events when asked to report their most severe life event (e.g. family conflict and serious illness). 


\section{Discussion}

Within a representative population-based sample, we assessed the prevalence of $\mathrm{AjD}$ according to a new stress response model [7], which is intended to overcome the former unsatisfactory conceptualisation. We used an abbreviated form of the AjD questionnaire, which used one or two essential symptom criteria per symptom group to diagnose $\mathrm{AjD}$ [15]. The reported prevalence rates of $1.3 \%$ (without meeting the impairment criterion) and $0.9 \%$ (meeting the impairment criterion) are consistent with previous findings $[12,16]$. No significant differences were found with respect to gender or age.

The Outcome of the Depression International Network (ODIN) found AjD prevalence rates of $0.2-1.0 \%$ in several European countries applying the strict exclusion criterion ("does not meet the criteria for another specific disorder") [12]. In a previous study applying the new stress response model, we found a prevalence of $2.3 \%$ in a general population sample of older individuals (65-96 years) [8]. The reported discrepancy in prevalence rates between older individuals from Germany (1.3\%) and Switzerland (2.3\%) may be explained by differences in how the target age ranges were defined (in the German subsample, individuals were required to be older than 60 , and the average age was 69 years; in Switzerland, they were required to be over 65 , and the average age was 74 years). In addition, the Swiss subsample exclusively comprised individuals living in urban areas. Finally, the Swiss study used a precursor version of the current $\mathrm{AjD}$ questionnaire. All the prevalence rates described lie far below the rates reported for atrisk samples. In psychosomatic clinical settings, AjD assessed by its conventional previous definition was found in up to $26 \%$ [17] of patients, as well as in $12 \%$ of patients from consultation-liaison psychiatry [4]. The results of this study demonstrate that the new conceptualisation of AjD does not lead to an overestimation of the disorder, even though the criterion to exclude other disorders was not applied. Although gender differences were found for some symptom clusters, no significant gender differences appeared for AjD diagnoses. However, the numeric frequency of $\mathrm{AD}$ was twice as high among women as in men, as in a previous study [12].

The following sections will discuss the impairment criterion and the time course of symptoms before considering validity aspects of the new AjD assessment concept. In this study, a difference in prevalence rates of $0.5 \%$ (which is nearly a third of the overall prevalence rate) was found between $\mathrm{AjD}$ with impairment (i.e. the criterion of clinical significance was fulfilled) versus without impairment. Note that the impairment criterion was similar to the other ratings, in that it was based on subjective reports rather than on clinical assessments. The impairment criterion is a component of the current psychiatric classification systems, but it has been previously questioned for certain diagnosis categories, such as anxiety disorders [18, 19]. We suggest either omitting the psychiatric impairment criterion in general, or adapting for AjD in particular ("significant deterioration in social and occupational functioning").

With the exception of the depressive subtype, the ICD restricts AjD subtypes to last for a maximum of 6 months; the DSM, however, distinguishes between acute ( $<6$ months) and chronic (more than 6 months) AjD. Clinicians indicate that they usually treat patients suffering from AjD for more than 6 months, sometimes more than 24 months (e.g. [20]). To our knowledge, no study has previously investigated the duration of $\mathrm{AjD}$. In the present study, participants were asked to indicate the duration of their symptoms, and they were provided with three possible time intervals: $0-1$ month, 1-6 months and 6-24 months. To summarise, for two-thirds of the symptoms, duration was indicated as 6-24 months. This result was not only true for the depressive subtype, but in most cases with mixed subtypes (mixed disturbance of emotions and conduct or mixed anxiety and depressed mood). Qualitative analyses of duration showed bereavement cases to constitute the largest portion of this group (26\%). For a certain subset of 6-24 months symptom cases (15\%), several stressor events were linked to conjointly cause $\mathrm{AjD}$ (e.g. family conflict with serious illness or moving). More research is required focusing on this time and course-related phenomena in $\mathrm{AjD}$.

The first aim of the study, estimating the prevalence of $\mathrm{AjD}$ in the general population, has to be discussed considering methodological aspects of the new AjD model. The conceptualisation of $\mathrm{AjD}$ as a stress response syndrome is currently being acknowledged by increasing numbers of experts in the field $[3,10]$. According to Strain and Friedman [10], one reason that there has been very little research on $\mathrm{AjD}$ is that $\mathrm{AjD}$ diagnostic criteria do not include specific symptoms. No recognised or standardised diagnostic instrument has yet been developed for epidemiological research or clinical trials on AjD. Furthermore, the authors point out that, in principle, such an instrument would include items tapping stressor-relatedness, mood, anxiety and behaviour. As demonstrated in this study, it is possible to draw face-valid and epidemiologically plausible conclusions with the help of the AjD questionnaire by Einsle et al. [15]. Further application of the theoretical concept of $\mathrm{AjD}$ and the derivation of an assessment tool will lead to the essential body of knowledge that will facilitate the qualified use of this diagnosis.

The present study has several shortcomings. One limitation is the use of self-report instruments rather than clinical interviews. Clinical interviews are considered more reliable for assessing clinical symptoms, although questionnaire studies are very common in epidemiological 
research. Furthermore, because of time constraints, we used a shortened version of the original AjD questionnaire. For a future screening tool, the regular process of standardisation should be carried out. Additionally, problems of recollection might bias investigations such as this in which the duration of symptoms and diagnosis are investigated with respect to a 2-year time period. Moreover, seriously ill persons might be underrepresented in the sample, as serious illness is expected to be associated with refusal of participation. Despite these constraints, the study reports an estimate of $\mathrm{AjD}$ prevalence that is not extremely high, even though this was the first time the exclusion criterion ("does not meet the criteria for another specific disorder") was not applied. The rate of $1.4 \%$ found in this study is comparable with the countryspecific estimations of $0.3-1.0 \%$ that were reported by the only other publication on population-based prevalence rates of AjD (European ODIN-multisite Study [12]).

\section{Conflict of interest None.}

\section{Appendix}

Adjustment disorders symptom clusters and symptom criteria assessed in the study.

Intrusions

- Repetitive thoughts

- Stressful thinking about it

- Constant reminders

- Unable to stop memories.

Avoidance

- Avoiding reminders

- Abolishing stressful thoughts

- Suppressing feelings

- Not talking about stressor.

Failure to adapt

- More difficulties concentrating

- Trouble sleeping since stressor

- Not keen to perform necessary tasks.

Depressive mood

- Low/sad

- Fewer enjoyable activities

- Feeling dispirited.

Anxiety

- Anxiety when thinking of stressor

- Fear of certain situations.
Impulse disturbance

- Quicker to lose one's temper

- More irritable

- Nervous and restless.

\section{References}

1. Baumeister H, Kufner K (2009) It is time to adjust the adjustment disorder category. Curr Opin Psychiatry 22:409-412

2. Baumeister H, Maercker A, Casey P (2009) Adjustment disorder with depressed mood: a critique of its DSM-IV and ICD-10 conceptualisations and recommendations for the future. Psychopathology 42:139-147

3. Semprini F, Fava GA, Sonino N (2010) The spectrum of adjustment disorders: too broad to be clinically helpful. CNS Spectr 15:382-388

4. Strain JJ, Smith GC, Hammer JS, McKenzie DP, Blumenfield M et al (1998) Adjustment disorder: a multisite study of its utilization and interventions in the consultation-liaison psychiatry setting. Gen Hosp Psychiatry 20:139-149

5. Gorschenek N, Schwab R, Eckert J (2008) Psychotherapy of adjustment disorders. Psychother Psychosom Med Psychol 58:200-207

6. vander Klink JJ, van Dijk FJ (2003) Dutch practice guidelines for managing adjustment disorders in occupational and primary health care. Scand J Work Environ Health 29:478-487

7. Maercker A, Einsle F, Köllner V (2007) Adjustment disorders as stress response syndromes: a new diagnostic concept and its exploration in a medical sample. Psychopathology 40:135-146

8. Maercker A, Forstmeier S, Enzler A, Krüsi G, Hörler E, Maier C, Ehlert U (2008) Adjustment disorders, posttraumatic stress disorder, and depressive disorders in old age: findings from a community survey. Compr Psychiatry 49:113-120

9. Dobricki M, Komproe IH, deJong TVM, Maercker A (2010) Adjustment disorders after severe life-events in four postconflict settings. Soc Psychiatry Psychiatr Epidemiol 45:39-46

10. Strain JJ, Friedman MJ (2011) Considering adjustment disorders as stress response syndromes for DSM-5. Depress Anxiety 28:818-823

11. Reed GM (2010) Toward ICD-11 Improving the Clinical Utility of WHO's International Classification of Mental Disorders. Prof Psychol Res Pract 41:457-464

12. Ayuso-Mateos JL, Vazquez-Barquero JL, Dowrick C, Lehtinen V, Dalgard OS, Casey P, Wilkinson C et al (2001) Depressive disorders in Europe: prevalence figures from the ODIN study. Br J Psychiatry 179:308-316

13. Foster P, Oxman T (1994) A descriptive study of adjustment disorder diagnoses in general hospital patients. Irish J Psychol Med 11:153-157

14. Greenberg WM, Rosenfeld DN, Ortega EA (1995) Adjustment disorder as an admission diagnosis. Am J Psychiatry 152:459-461

15. Einsle F, Köllner V, Dannemann S, Maercker A (2010) Development and validation of a self-report for the assessment of adjustment disorders. Psychol Health Med 15:584-595

16. Casey P, Maracy M et al (2006) Can adjustment disorder and depressive episode be distinguished? Results from ODIN. J Affect Disord 92:291-297

17. Despland JN, Monod L, Ferrero F (1995) Clinical relevance of adjustment disorder in DSM-III-4 and DSM-IV. Compr Psychiatry $36: 454-460$ 
18. Grenier S, Preville M et al (2011) The impact of DSM-IV symptom and clinical significance criteria on the prevalence estimates of subthreshold and threshold anxiety in the older adult population. Am J Geriatr Psychiatry 19:316-326

19. Zimmermann G, Pin MA, Krenz S et al (2004) Prevalence of social phobia in a clinical sample of drug dependent patients. J Affect Disord 83:83-87
20. Rätzel-Kürzdörfer W, Franke C, Wolfersdorf M (2003) Casuistry of a long duration depressive adjustment disorder. Diagnosis of efficiency and psychotherapeutic treatment of adjustment disorder. Psychiatr Prax 30:85-87 